\title{
Successfully aging elderly (SAE): A short overview of some important aspects of successful aging
}

\author{
Eystein Stordal $^{1,2}$, Ingunn Bosnes ${ }^{1}$, Ole Bosnes ${ }^{1}$, Elisabeth Bratland Romuld ${ }^{1}$ and Ove Almkvist ${ }^{3,4}$ \\ 1) Department of Psychiatry, Hospital Namsos, Namsos, Norway \\ 2) Department of Neuroscience, Faculty of Medicine, Norwegian University of Science and Technology, \\ Trondheim, Norway \\ 3) Department of Psychology, Stockholm University, Stockholm, Sweden \\ 4) Department of Neurobiology Care Sciences and Society, Karolinska Institutet, Stockholm, Sweden \\ Correspondence: Eystein Stordal, Department of Psychiatry, Hospital Namsos, N-7800 Namsos, Norway \\ E-mail: eystein.stordal@hnt.no Telephone: +47 74215400
}

\begin{abstract}
Whether one falls into the category of successfully aging elderly (SAE) is generally determined by biological, medical, psychological, and cognitive factors. SAE, pathological aging and usual aging, are the three subgroups presented in the seminal science paper by Rowe \& Kahn in 1987. SAE is currently vaguely defined as being free of disease, having preserved cognitive function and an active life, but a more detailed definition is lacking. As a result, the research on SAE is heterogeneous and hard to summarize. Nevertheless, it is clear that genetics, health, basic aging mechanisms, brain changes, cognition early in life, education level, lifestyle factors, subjective factors, the availability of societal health care, environmental factors, and any interaction between all these variables, are important. There are also methodological difficulties associated with studies of causal relationships across the lifespan. Obtaining a detailed understanding of SAE research will be a challenging task for future researchers.
\end{abstract}

\section{INTRODUCTION}

Most of the epidemiological and clinical research carried out in elderly populations so far has focused on those with diseases and functional impairment. Less research has been done on the part of the population that reaches old age without disease or functional impairment. In disease research, each disorder has predefined criteria to determine its presence. This is not the case when it comes to the category of SAE, and it has been suggested that health conditions could be used to differentiate between subgroups of elderly populations, and that disease-free individuals could represent the SAE (1).

The present paper gives a brief overview of SAE research, presented in three main sections: biological and medical aspects; psychological and social aspects; and cognitive aspects, all of which are either known, or thought to be important for cognitive function in SAE. Although our main focus will be the psychological and cognitive aspects of SAE, this does not mean that we consider the biological, medical and social aspects to be less important. We propose that future research should employ an extended definition of SAE, where the focus is more on the stability of cognitive function, or other health aspects, rather than on level of performance which is currently used to define SAE.

\section{The concept of successful aging}

The concept of successful aging (SAE), sometimes also called optimal health or superaging, was introduced by Rowe \& Kahn in 1987 (2). Essentially, SAE represents neither pathological aging, e.g., dementia, nor usual aging, e.g., a gradual decline of psychological and bio- logical functioning. Instead SAE represents a kind of aging associated with a low probability of disease or disability, high cognitive and physical function, as well as active engagement with life, including maintenance of autonomy $(2,3)$. Other researchers have proposed a more general definition, made up of six categories: no physical disability over the age of 75 as rated by a physician, good self-rated health status, length of undisabled life, good mental health, objective social support, and self-rated life satisfaction in eight domains, namely marriage, income-related work, children, friendship and social contacts, hobbies, community service activities, religion and recreation/sports (4). More restricted definitions have also been proposed regarding cognitive performance, for instance a classification as SAE when the cognitive performance of 70 - to 85 -year-olds is above the mean for 50 to 65 -year-olds (5).

Figure 1 shows that the frequency of SAE is thought to remain fairly constant until late in life, while there is a steady fall in the frequency of usual aging throughout life, and a sharp rise in the frequency of pathological aging after approximately 80 years. For all three categories of aging, terminal cognitive decline is expected to occur close to the end of life (6). This description of prevalence of the categories of aging across time must be viewed as a tentative model of cognitive aging until more data on prevalence and incidence are reported.

An illustration of the diversity of elderly non-demented individuals can be found in the publications on the $\mathrm{H} 70$ (age 70 was an inclusion criteria) epidemiological study carried out in Göteborg, Sweden (7), in which five categories of elderly individuals were empirically 




Figure 1. A hypothetical illustration of the prevalence of pathological aging, usual aging and successful aging between age 55 and 95 years.

found, based on functional capacity, subjective wellbeing, social contacts, cognitive function and physical health status. The individuals were categorized as (i) high in all five measures $(25 \%$; corresponding to SAE), which was associated with high education level and the longest survival, (ii) low in all five measures, (iii) low in cognition only, or (iv) high in one of either cognition, social contacts, or subjective well-being, or (v) low in one of either physical health status, social contacts, or subjective well-being. Hypothetically, groups (ii)-(v) could represent various subgroups of usual aging, when compared to the Rowe \& Kahn criteria, and therefore the H70 study could be considered a demonstration of the effect of the dynamic interaction between various aspects on the aging process. Although SAE has stimulated research on factors that are important for healthy aging, it still has a vague definition, and consequently a lot of conflicting empirical findings.

\section{Overview of SAE and causal factors}

Hypothetically, pathological aging, usual aging and successful aging are related to the interaction of a number of possible causative factors. These factors concern biological, medical, lifestyle and environmental factors, which are differentially important in these three aging categories. All individuals are affected by innate biological and medical factors, such as immune system issues, but not all are affected by disease. Environmental and lifestyle factors may vary in their influence, depending on the individual, and their environment and lifestyle. In this way, the pattern and interaction of causative factors may determine the specific life course of each individual.

In pathological aging, the combined influence of biological factors, disease, lifestyle and environmental factors may cause pronounced cognitive decline, disability, and lowered quality of life. In contrast, the influence of negative causative factors on SAE individu- als is less, perhaps because they have genes related to longevity and freedom from disease and/or they have a favorable lifestyle. This scenario illustrates the possible relationship between the three categories of aging and the interaction with factors that have an impact on aging.

\section{BIOLOGICAL AND MEDICAL ASPECTS}

\section{Disease and disability}

Rowe and Kahn $(2,3)$ listed avoidance of disease and disability and high cognitive and physical function as two of the three main components of successful aging. In this setting, the term "avoidance of disease" refers not only to the absence of mental or physical disease, but also to the absence of risk factors for disease. All chronic physical diseases that cause functional impairment, the most important of which are diseases that influence brain function, are exclusion criteria for SAE. Degenerative neurological disorders, such as Alzheimer's disease, Parkinson's disease and multiple sclerosis influence brain function directly, while cardiovascular disorders and stroke influence brain function indirectly through vascular changes in the brain. Other common chronic disorders such as musculoskeletal disorders cause impaired physical function due to reduced movement and pain. Fractures, the most common being hip fractures, are another physical condition that leads to reduced physical function, and in many cases also to cognitive impairment. Furthermore, reduced sight and hearing are common factors that can lead to inactivity and reduced cognitive stimulation and function. Persons suffering from serious psychiatric disorders like schizophrenia, psychotic disorders and bipolar disorders are also excluded as SAE. In addition, elderly persons with minor psychiatric diagnoses, such as depression and anxiety disorders, who have symptoms that influence quality of life and cognitive function, are excluded.

\section{Possible theories about the biological and medical model of $S A E$}

Both genetic and environmental factors are important for SAE. Much research has been done in the last two decades on genetics and basic biological phenomena that may affect the aging process.

\section{Genetic factors}

Research on the relationship between cognitive function and genetics indicates that a part of the variation in global cognition in adulthood can be linked to genetic factors (8). Genetics may also influence longevity (9) and disease occurrence. The relative importance of genetic factors as a cause of decreased cognitive function seems to diminish during the aging process (4). The genetic factors that are most important for SAE seem to be those related to cardiovascular health, inflammatory processes, and fundamental cell conditions.

An example of the current studies on genetics and cognition is a study by Nilsson and colleagues based 
on 4200 participants in the Betula study. The results showed that among persons 70 years or older, three genes (APOE, COMT, and KIBRA) were related to SAE and good performance on cognitive tests (10). Other studies have added BDNF to the list of genes that may be related to cognitive function in SAE (11). It is interesting to note that, at least for health status, the importance of genetic factors decreased with advancing age (3), while the importance of nongenetic factors increased, which has implications for health care and society, as prevention programs that target the ederly may be of greater value than previously thought.

\section{Other basic biological factors}

In basic research several factors that influence the metabolic function of brain cells and neuronal membranes can lead to cell death and influence the aging process. Oxidative stress, decline in mitochondrial function, reduced ability to detoxify free radicals, accumulation of potentially injurious proteins and the effect of telomeres are some of these factors (12-14). Changes in neurotransmitter systems, such as a decline in brain dopamine activity is another possible factor (15). Other researchers have pointed out that the immune system plays a role in aging (16).

\section{Brain characteristics and aging}

In recent studies on brain characteristics in normal aging $(17,18)$, age-related shrinkage of brain volume in SAE and usual aging was reported, as well as an increase in white matter pathology. These changes were paralleled by changes in cognitive performance. Functional MRI studies also demonstrated that age-related changes in brain activity, such as reduced signals, change in regional patterns and additional recruitment of significantly active brain regions. In SAE, it has been shown that preserved hippocampal activation during encoding is linked to good episodic memory performance (19).

\section{Cognitive function and training}

Intervention studies have reported that aerobic physical training increase cardiorespiratory fitness, as well as cognitive function in older adults (20). In some of these studies both behavioral improvement and changes in brain characteristics have been reported. There are also many cognitive/brain training programs on the market, but few of these programs present evidence on their efficacy in real life. However, research studies on cognitive training in elderly individuals have reported clear positive effects on daily life, although the effects were not significant within the training program (20).

Finally it is noteworthy that there is a parallel between human studies on training and stimulation intervention and animal studies on the positive cognitive effect of enriched environment. Conversely there is also a parallel between studies on the negative effect of restricted stimulation on the development of the central nervous system (21).

\section{PSYCHOLOGICAL AND SOCIAL ASPECTS}

The main reason for the growing focus on cognitive function in old age, especially during the last 20 or 30 years, is that it has a tremendous impact on our possibility to maintain independence and a high quality of life during aging. In individuals that fulfill the criteria of SAE, cognitive function is well maintained in old age, in contrast both to pathological aging and usual aging. In pathological aging the changes in cognitive function are much more pronounced than in usual aging, due to a number of diseases (e.g., Alzheimer's disease and cerebrovascular diseases) that cause irreversible brain destruction.

\section{Lifestyle}

In recent years, epidemiological research on lifestyle factors that may promote maintained cognitive function has increased tremendously (20). Four key areas have been suggested as promising: high cognitive activity, high physical activity, active social engagement and good nutrition. Interestingly, the same factors found at low levels have been reported as possible risk factors for pathological aging and dementia $(4,20,21)$.

Longitudinal studies on the relationship between cognitive function and health status has shown that education level, as well as related concepts of mastery and control and self-efficacy, are essential factors, in addition to physical activity/training and measures of cardiovascular status (21). On the other hand, low education level seems to be a risk factor for dementia development.

\section{Self-rated health status and SAE}

Self-rated health status is usually relatively high and stable across age groups (around $80 \%$ of subjects rate themselves as in good health) in spite of an increasing frequency of disease, from very low in a person's 20 s to high in a person's 80s (10). In another study good self-rated health status was reported by half of an elderly population (23).

The correlation between self-rated health status and health indicators, as well as memory performance, has generally been found to be very low, except for medication and contacts with health care, where significant correlations were found (24). Contrary to this pattern, a number of health indicators were significantly associated with episodic memory performance (10). One problem with such correlation studies is that statistical significance is not always a good marker of clinical significance.

The focus of psychosocial definitions includes previous, present and future expectation of life satisfaction, well-being and participation in social life, while the biomedical status may be less important. A positive attitude to life and effective coping with present circumstances, not physical health, seem to be the essential factors. Well-being may even be experienced in 
the presence of disease or disability. In a random national British survey of SAE, 75\% of individuals aged 50 or more reported a self-rated status of very well (25) for both health and various psychosocial factors. This fact may have far reaching implications both for the health care authorities as well as government-run social intervention programs. In the future it may well become an ethical dilemma as to where to put your priorities, efforts and money, into successful biomedical health or psychosocial satisfaction?

\section{COGNITIVE ASPECTS}

Pathological aging increases from almost nil at age 65 to $50-75 \%$ closer to 100 years of age (26). In contrast to usual aging and pathological aging, in SAE cognitive function remains in effect unchanged, or changed to only a minor degree, until the last part of life (4), when terminal decline (clear drop in cognitive function during the last years of life) may occur affecting cognitive domains in general (6). At ages 70 and older, SAE estimates of $10 \%$ have been reported based on memory performance $(27,28)$, with a probable decrease to only a few percent at high age, according to biomedical definitions (23), though the percentage varies substantially depending on the study criteria and methods used.

In previous research, cognitive function has been studied by less demanding measures, such as the MiniMental Status Examination, as well as comprehensive sets of specific neuropsychological tests. This variation on cognitive demands will have an impact on the results. By using a demanding set of tests (23 tests focused on memory), the epidemiological Betula study (5) identified $8 \%$ of its non-demented 70-85-year-old participants as SAE when the criterion for performance was set above the mean level for middle-aged individuals (50-65 years old). About one-third of these individuals remained in the SAE category 5 years later, while the other two-thirds had declined to the status of non-SAE individuals. At the same time, a small proportion of individuals classified as non-SAE at baseline improved in cognition after a 5-year follow-up $(2 \%)$ and were reclassified as SAE individuals. In that study, the main predictor of SAE was high education level. The idea that maintained cognitive function may be associated with higher education level has been related to the concept of cognitive reserve (29), i.e., the idea that individuals who had a high previous cognitive capacity can process tasks well, even after brain pathology has begun.

An interesting example of SAE is the life of Madame Calment (30). This woman performed comparably to people in their 80 s on language and memory tests at over 100 years of age. She had no signs of depression, and a good sense of humor; upon CT examination, it was observed that her brain had been relatively spared. Both her paternal and maternal ancestors had a long life. She did not recall any serious illness during her life and lived independently until the age of 115 years.

\section{Cognition in usual aging}

In usual aging, there is a decline in some cognitive domains, typically processing speed, episodic memory and executive functions, while other domains, typically semantic knowledge, procedural memory, information and reasoning, are largely unaffected, or even better through the life span $(27,28,31,32)$. The onset of changes may occur in middle age or later $(28,33)$. The cognitive functions most vulnerable to aging (and disease) have sometimes been summarized in the term "fluid functions", while the functions more immune to change are sometimes referred to as "crystallized functions" (34).

When presenting data on age-related changes, it is important to base descriptions on longitudinal data corrected for inter-individual confounding effects (e.g., education level, manifest and latent disease, socioeconomic status, etc.), as well as generational cohort effects, rather than cross-sectional data, which usually give an exaggerated view of the negative age effect on cognitive function over time $(28,33,35)$. It is also important to consider the possible increasing interindividual variation in cognitive function over time.

\section{Cognitive stability}

It is well known that there is a high degree of cognitive stability from young age to advanced age. For example a Scottish study covering 66 years found a correlation of $r=0.73$ in global cognition (36).

Seim published some pioneering work in which a group of 11-year-old Norwegian preadolescents were tested repeatedly until age 80 years, and which indicated that personality and cognition generally do not change much throughout life (37). More recently the results regarding cognitive stability were replicated in the Lothian Birth Cohorts, which covered global intelligence between 11 and 70-87 years of age (38). Childhood global cognitive ability was the most powerful predictor of global cognitive ability in late life. Other predictors of late life cognitive ability were education level and gender, but the effect of these factors was minimal. Interestingly, none of the predictors had a significant impact on changes in cognitive function in late life. So it is seems to be important to differentiate between the effect on the level of cognitive function and on changes in cognitive function in late life. Hypothetically genetics are important to global intelligence both in childhood and in late life, while disease is important for change in ability.

Is an expanded definition of SAE needed?

The current definitions of SAE exclude nearly $90 \%$ of the elderly population, which poses the risk that research based on this very selected group cannot be applied to the category of usual aging, not to mention the category of pathological aging. Our research group believes that a broader definition of SAE, based more on the stability of cognitive function than on the level of functioning, on which the current definitions are 
heavily based, is needed. We also believe that a broader definition is in line with current clinical work, and will probably initiate more research relevant to the development of both preventive and treatment strategies for older people in general.

\section{Findings from the Older Norwegian Normative Study (ONNS) in Nord-Trondelag}

A presumably healthy sample of 180 individuals aged 55-89 years were randomly selected from the HUNT 3 Research Center database $(39,40)$. Out of these, a total of 122 individuals were consecutively recruited to a normative study of memory and intelligence, the ONNS, and were tested with the Wechsler Memory Scale (WMS-III) and the Wechsler Adult Intelligence Scale (WAIS-III). Inclusion and exclusion criteria used in the standardization studies of memory and intelligence in the USA (WAIS-III/WMS-III. Technical Manual, 1997) were followed as closely as possible, rendering a sample in good mental and physical health. In addition to this procedure, each individual invited was interviewed before WAIS-III/WMS-III, when they were asked questions about any mental or physical diseases affecting their cognition, or that might make it difficult to accomplish the testing. It can thus be argued that the 122 participants included are probably a representative sample of the healthy population between 55 and 89 years in Nord-Trøndelag. The test results also showed a distribution that was close to normal (although the number of participants at both the very low and the very high scoring range was somewhat small), which adds support to the argument that the sample is representative (41). As a previous study (39) found that the population of NordTrøndelag is largely representative of the Norwegian population, this means that the results from ONNS probably are representative of the older Norwegian population. Individuals from this study can be further selected according to specific criteria to define a group of SAE, based on information collected before testing, test results and data from the HUNT studies.

\section{FUTURE PLANS REGARDING STUDIES OF SUCCESSFUL AGING}

New longitudinal studies are needed to study factors that influence SAE. Our research group has studies planned that will use the healthy individuals in the ONNS study of Nord-Trøndelag as an endpoint, and longitudinal data from the three HUNT studies (19841986, 1995-1997, 2006-2008) as predicting factors. Further we will study SAE and depression, headache, metabolic syndrome, physical activity and sleeping patterns.

\section{REFERENCES}

1. Depp CA, Jeste DV. Definitions and predictors of successful aging: A comprehensive review of larger quantitative studies. Am J Geriatr Psychiatry 2006; 14: 6-20.

2. Rowe JW, Kahn RL. Human aging: usual and successful. Science 1987; 237: 143-9.

3. Rowe JW, Kahn RL. Successful aging. Gerontologist 1997; 37: 433-40.

4. Depp CA, Harmell A, Vahia IV. Successful cognitive aging. Curr Top Behav Neurosci 2012; 10: 35-50.

5. Habib R, Nyberg 1, Nilsson LG. Cognitive and non-cognitive factors contributiong to the longitudinal identification of successful older adults in the Betula study. Aging Neuropsychol Cogn 2007; 14: $257-73$.

6. Wilson RS, Beckett LA, Bienias JL, et al. Terminal decline in cognition. Neurology 2003; 60: 1782-7.

7. Maxson PJ, Berg S, McClearn G. Multidimensional patterns of aging in 70 -year-olds: survival differences. $J$ Aging Health 1996; 8: 320-33.

8. Finkel D, Pedersen NL, Plomin R, McClearn GE. Longitudinal and cross-sectional twin data on cognitive abilities in adulthood the Swedish adoption/twin study of aging. Dev Psychol 1998; 34: 1400-13.

9. Lopez LM, Harris SE, Luciano M, et al. Evolutionary conserved longevity genes and human cognitive abilities in elderly cohorts. Eur J Hum Genet 2012; 20: 341-7.

10. Nilsson LG, Bäckman L, Erngrund K, et al. The Betula prosespective cohort study: memory, health, and aging. Aging Neuropsychol Cogn 1997; 4: 1-32.

11. Li SC, Lindenberger U, Bäckman. Dopaminergic modulation of cognition across the life span. Neurosci Biobehav Rev 2010; 34: 625-30.

12. Hipkiss AR. Accumulation of altered proteins and ageing: causes and effects. Exp Gerontol 2006; 41: 464-73.

13. Terman A Gustafsson B, Brunk UT. Mitochondrial damage and intralysosomal degradation in cellular aging. Mol Aspects Med 2006; 27: 471-82.

14. Aviv A, Levy D, Mangel M. Growth, telomere dynamics and sussessful and unsuccessful human aging. Mech Ageing Dev 2003; 124: 829-37.

15. Volkow ND, Gur RC, Wang GJ, et al. Association between decline in brain dopamine activity with age and cognitive and motor impairment in healthy individuals. Am J Psychiatry 1998; 155: 344-9.

16. Alonso-Fernández P, De la Fuente M. Role of the immune system in aging and longevity. Curr Aging Sci 2011; 4: 78-100. 
17. Fjell AM, Walhovd KB, Fennema-Notestine CF, et al. One-year brain atrophy evident in healthy aging. $J$ Neurosci 2009; 29: 15223-31.

18. Raz N, Lindenberger U, Rodrigue KM, et al. Regional brain changes in aging healthy adults: General trends, individual differences and modifiers. Cerebral Cortex 2005; 15: 1676-89.

19. Düzel E, Schütze H, Yonelinas AP, Heinze HJ. Functional phenotyping of successful aging in long-term memory: Preserved performance in the absence of neural compensation. Hippocampus 2011; 21: 803-14.

20. Daffner KR. Promoting successful cognitive aging: a comprehensive review. J Alzheimers Dis 2010; 19: 1101-22.

21. Hubel DH Wiesel TN. The period of susceptibility to the physiological effects of unilateral eye closure in kittens. J Physiol 1970; 206: 419-36.

22. Albert MS, Jones K, Savage CR, et al. Predictors of cognitive change in older persons: MacArthur studies of successful aging. Psychol Aging 1995; 10: 578-89.

23. Strawbridge WJ, Wallhagen MI, Cohen RD. Successful aging and well-being: self-rated compared with Rowe and Kahn. Gerontologist 2002; 42: 727-33.

24. Vestergren P, Rønnlund M, Nyberg L, et al. Development of the Cognitive Dysfunction Questionnaire (CDQ) in a population based sample. Scand J Psychol 2011; 52: 218-28.

25. Bowling A, Dieppe P. What is successful aging and who should define it? BMJ 2005; 331: 24-31.

26. Ferri CP, Prince M, Brayne C, et al. Global prevalence of dementia: a Delphi consensus study. Lancet 2005; 366: $2112-7$.

27. Nyberg L, Maitland SB, Rönnlund M, et al. Selective adult age differences in an age-invariant model of declarative memory. Psychol Aging 2003; 18: 149-60.

28. Nyberg L, Lövdén M, Riklund K, et al. Memory aging and brain maintenance. Trends Cogn Sci 2012; 16: 292-305.

29. Stern Y. Cognitive reserve. Neuropsychologia 2009; 47: 2015-28.

30. Ritchie K. Mental status examination of an exeptional case of longevity: J.C. aged 118 years. Br J Psychiatry 1995; 166: 229-35.

31. Bäckman L, Wahlin $\AA$, Small BJ, et al. Cognitive functioning in aging and dementia: the Kungsholmen project. Aging Neuropsychol Cogn 2004; 11: 212-44.

32. Deary IJ, Corley J, Gow AJ, et al. Age-associated cognitive decline. Br Med Bull 2009; 92: 135-52.

33. Singh-Manoux A, Kivimaki M, Glymour M, et al. Timing of onset of cognitive decline: results from Whitehall II prospective cohort study. BMJ 2011; 344: d7622.

34. Horn JL, Cattell EB. Age differences in fluid and crystallized intelligence. Acta Psychol 1967; 26: 107-29.

35. Rönnlund M, Nyberg L, Bäckman L, Nilsson LG. Stability, growth and decline in adult life span development of declarative memory: cross-sectional and longitudinal data from a population-based study. Psychol Aging 2005; 20: 3-18.

36. Crawford JR, Deary IJ, Starr J, Whalley LJ. The NART as an index of prior intellectueal functioning: a retrospective validity study covering a 66-year interval. Psychol Med 2001; 31: 451-8.

37. Seim S. Tenåringen blir pensjonist. NOVA rapport 23, Norsk institutt for forsking, velferd og aldring, 1997.

38. Gow AJ, Johnson W, Pattie A, et al. Stability and change in intelligence from age 11 to ages 70, 79, and 87: the Lothian Birth Cohorts of 1921 and 1936. Psychol Aging 2011; 26: 232-40.

39. Holmen J, Midthjell K, Krüger Ø, et al. The Nord-Trøndelag Health Study 1995-97 (HUNT2): objectives, contents, method and participation. Norsk Epidemiologi 2003; 13: 19-32.

40. http://www.ntnu.no/hunt.

41. Bosnes O, Troland K. Wechsler Memory Scale-III og Wechsler Adult Intelligence Scale-III utprøvd i et utvalg av HUNT 3-populationen [WMS-III and WAIS-III evaluated in a sample of HUNT 3]. Tidskr nor psykologforen 2012; 49: 462-7. 